University of New Orleans

ScholarWorks@UNO

Physics Faculty Publications

Department of Physics

1989

\title{
On the size distribution of newly formed grains in red supergiant atmospheres
}

C Gregory Seab

University of New Orleans

Theodore P. Snow

Follow this and additional works at: https://scholarworks.uno.edu/phys_facpubs

Part of the Astrophysics and Astronomy Commons, and the Physics Commons

\section{Recommended Citation}

Astrophys. J. 347479 (1989)

This Article is brought to you for free and open access by the Department of Physics at ScholarWorks@UNO. It has been accepted for inclusion in Physics Faculty Publications by an authorized administrator of ScholarWorks@UNO.

For more information, please contact scholarworks@uno.edu. 
THE ASTROPHYSICAL JOURNAL, 347:479-482, 1989 December 1

(C) 1989. The American Astronomical Society. All rights reserved. Printed in U.S.A.

\title{
ON THE SIZE DISTRIBUTION OF NEWLY FORMED GRAINS IN RED SUPERGIANT ATMOSPHERES
}

\author{
C. Gregory Seab \\ Department of Physics, University of New Orleans \\ AND \\ THEODORE P. SNOW ${ }^{1}$ \\ Center for Astrophysics and Space Astronomy, University of Colorado \\ Received 1988 July 18; accepted 1989 June 8
}

\begin{abstract}
Theoretical ultraviolet extinction curves have been calculated for comparison with observed curves for circumstellar dust in $\mathbf{M}$ supergiants. The theoretical curves assume a silicate grain composition, because silicate grains are expected in the oxygen-rich environments that are observed. Calculations were performed with and without the inclusion of scattering into the beam, with largely similar results. A comparison of the computed curves with the observed ultraviolet extinction curve for circumstellar dust in $\alpha$ Scorpii indicates that the size distribution of the circumstellar grains must cut off near $800 \AA$; that is, there are few or no grains smaller than this. Our conclusion is that smaller interstellar silicate grains, where they exist, must come from other sources such as grain fragmentation in shocks.
\end{abstract}

Subject headings: interstellar; grains - stars: atmospheres — stars: circumstellar shells — stars: late-type — ultraviolet: spectra

\section{INTRODUCTION}

A variety of evidence suggests that the general interstellar medium contains dust grains smaller than those responsible for visible-wavelength extinction and polarization. The evidence for smaller grains includes the rise of extinction toward short wavelengths in the ultraviolet (e.g., Witt 1973) which indicates the presence of grains as small as $500 \AA$ or less; the general fit of scattering models to the observed extinction, which requires small grains (e.g., Mathis, Rumpl, and Nordsieck 1977; Draine and Lee 1984); and the recent infrared indicators that the general size distribution may extend down to grains of only a few tens of angstroms in size (Sellgren 1984; Sellgren, Werner, and Dinerstein 1983).

If very small grains are present, they may play important roles in galactic and interstellar medium evolution. For example, if they are as numerous as would be expected from extrapolation of the standard grain-size distribution $\left(n \propto a^{-3.5}\right.$, where $a$ is grain radius; Mathis, Rumpl, and Nordsieck 1977), they should dominate the surface area available for the accretion of grain mantles in gas-grain collisions and might therefore govern interstellar depletions as well as ionization in dark clouds (Draine and Sutin 1987) and photoelectric heating of diffuse interstellar gas (Jura 1976). It is therefore important to understand the nature, number, and evolution of these grains.

Small grains can be the product of grain formation or they can be fragments of grains that are broken apart, for example, in interstellar shocks. Recent theoretical calculations indicate that the time scale for a typical grain to undergo shocks is an order of magnitude shorter than the time scale for formation and injection into the interstellar medium (Seab and Shull 1983; McKee et al. 1987; Seab 1987), suggesting that the observed size distribution must be the result of shock pro-

\footnotetext{
${ }^{1}$ Guest Investigator with the International Ultraviolet Explorer, sponsored by the National Aeronautics and Space Administration.
}

cessing rather than grain formation. Some calculations of grain-formation processes (e.g., Hasegawa 1984) support this, indicating that typically grains produced in red giant and supergiant atmospheres grow to several hundred angstroms before injection into the interstellar medium, while others (e.g., Gail and Sedlmayr 1986) predict small sizes for newly formed circumstellar grains. Thus theoretical models of grain formation leave open the question of whether the very small grains known to exist in the diffuse interstellar medium are formed by condensation in circumstellar environments or by shock fragmentation of larger grains.

In this paper we model observed circumstellar extinction curves, using scattering theory and plausible grain compositions. The observations are described in the next section, followed by a description of the calculations ( $\S$ III), and a discussion of the results and their implications ( $§$ IV).

\section{OBSERVATIONAL MATERIAL}

We have identified a number of binary systems containing an $\mathrm{M}$ supergiant and a hot ( $\mathrm{B}$ or $\mathrm{A}$ ) main-sequence companion. The most prominent of these is Antares ( $\alpha \mathrm{Sco}$ ), consisting of an M1 Ia primary and a B2 IV secondary (for a discussion of the secondary's spectral classification, see Snow et al. 1987). For $\alpha$ Sco and several other similar systems, it has been possible to make ultraviolet observations of the hot companion as a probe of the properties of the circumstellar dust being formed in the extended atmosphere of the red supergiant (Buss and Snow 1988). The primary products are ultraviolet extinction curves for the circumstellar dust, although some information on gas-phase depletions may also be derived.

There are many difficulties in the analysis, caused by foreground interstellar dust contributions to the extinction, but in cases where these difficulties could be resolved, the results have been quite consistent. The circumstellar extinction curves show no $2175 \AA$ bump, and no far-ultraviolet rise. The lack of a 2175 
$\AA$ bump may be attributed to the presumed lack of carbonaceous grains in these oxygen-rich environments, since most theories of the origin of the bump suggest that it arises in carbonaceous grains of some type (see, for example, the review by Savage and Mathis 1979 and the recent discussions by Hecht 1986 and Sakata et al. 1984). The lack of a far-ultraviolet rise is less easily explained as a grain-composition effect, however, so we decided to explore its implications for the grain-size distribution.

\section{THEORETICAL CALCULATIONS}

\section{a) Without Scattering into the Beam}

For our first attempt to see whether the flat far-UV extinction curve constrains the grain-size distribution, we undertook extinction curve calculations in which scattering of photons into the IUE aperture was neglected. This simplified case could be computed readily, though for a complete answer it proved necessary later to include scattering, as discussed in the next subsection.

A series of extinction curve calculations were carried out using a variety of silicate grain-size distributions. Optical constants for "astronomical silicate" have been taken from Draine and Lee (1984) and Draine (1985), including minor corrections from Draine (1987). Scattering cross sections were calculated using standard Mie theory for homogeneous spherical particles (van de Hulst 1957). Graphite grains are not included in the calculations because the oxygen-rich environment of stars like $\alpha$ Sco are expected to produce silicate grains but not graphite or other carbonaceous grains.

Grain extinction calculations were done assuming two different types of grain-size distributions. First, extinction curves were calculated assuming all grains were the same size. Values between $100 \AA$ and $2500 \AA$ were used for the radius. Second, a Mathis, Rumpl, and Nordsieck (1977) type of power-law distribution was used, which has the number density of grains $d n$ given by

$$
d n \propto a^{-3.5} d a, \quad a_{\min }<a<a_{\max } .
$$

For all power-law calculations, the value of $a_{\max }$ was fixed at $2500 \AA$, while the $a_{\text {min }}$ value was varied in logarithmic steps from the $100 \AA$ value appropriate to the normal interstellar medium up to the maximum of $2500 \AA$. Results for the powerlaw type distribution are shown in Figure 1 for $a_{\text {min }}$ values between $250 \AA$ and $1500 \AA$. Extinction curves for the singlesized grains are not shown, but lead to the same conclusions. A more general discussion of the results of varying the size limits in a power-law distribution is given by Mathis and Wallenhorst (1981).

\section{b) Inclusion of Scattering into the Beam}

In order to incorporate the possibility that some photons are scattered into the IUE aperture, we adopt the formalism of Code (1973), which solves the two-stream radiative transfer problem for a spherically symmetric circumstellar nebula entirely within the beam. While this situation does not apply directly to the $\alpha$ Sco system, the results should nevertheless indicate the direction of change when circumstellar scattering is included. A full solution would necessitate a Monte Carlo simulation of a UV source embedded off-center in an approximately spherical nebula centered on the $M$ star. As we discuss below, the results of using the Code approximation give us confidence that a more detailed calculation would not produce significantly different conclusions.

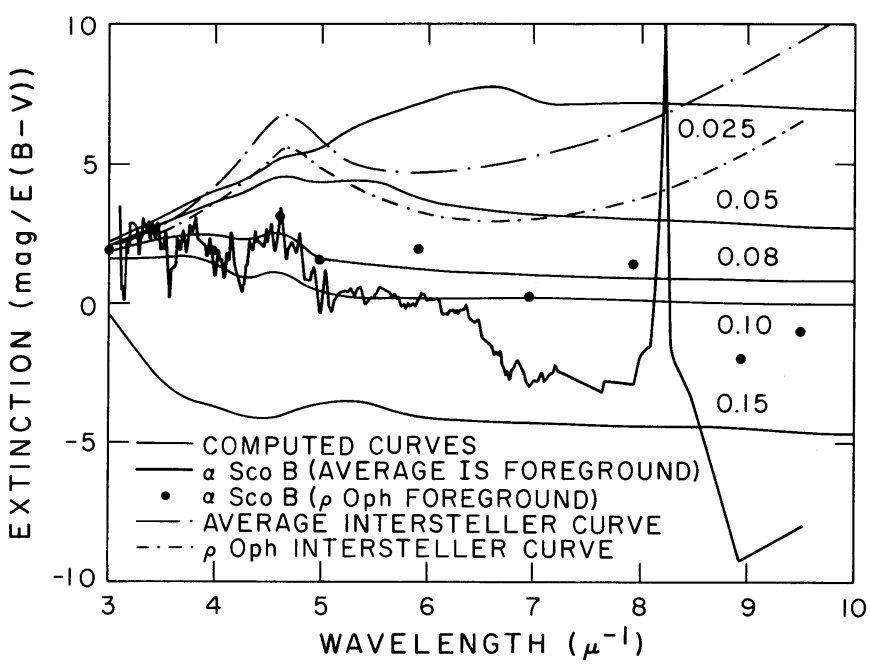

FIG. 1.-Comparison of computed curves with circumstellar extinction in $\alpha$ Sco. The flatness of the observed curve is inconsistent with the lack of a rise toward short wavelengths in all computed curves for minimum grain size $a_{\min }<800 \AA$. The $\alpha$ Sco curve from Snow et al. (1987) was based on subtraction of an average foreground interstellar extinction curve; we have estimated (dots) how this would be changed by adopting a relatively flat far-UV curve to represent the foreground extinction, as appropriate for the $\rho$ Ophiuchi cloud. The spike in the $\alpha$ Sco curve near $8 \mu \mathrm{m}^{-1}$ is due to Ly $\alpha$ absorption.

Code (1973) gives the emergent flux from the nebula plus the central star relative to that from the star alone as

$$
\frac{L}{L^{*}}=\frac{2}{(1+\zeta) e^{\xi \tau+}(1-\zeta) e^{-\xi \tau}}
$$

where

$$
\zeta=\sqrt{(1-\omega) /(1-\omega g)}
$$

and

$$
\xi=\sqrt{(1-\omega)(1-\omega g)},
$$

where $\omega$ is the grain albedo, $g$ is the forward-scattering parameter, and $\tau$ is the optical depth at $V$ to the star. This equation was used to determine an "effective extinction" which will be less than the expected total extinction by the amount of scattered light included in the beam.

The grain models used are the same as in the previous subsection. The total grain albedo and forward-scattering parameter are calculated from the optical properties of the "astronomical silicate" grain material. We addressed the grain optical depth by two different approaches. First, a selfconsistent solution of equation (1) was done by setting the effective extinction at $B-V$ (including scattering effects) equal to the observed $E(B-V)=0.15$ (Snow et al. 1987) and numerically solving for the required $\tau$. For all grain models, this procedure gave a result requiring more grain material than expected in the line of sight from the total hydrogen column density of hydrogen with a normal heavy element abundance and depletion. The resulting effective $E(B-V)$ is low compared to the observed value; we take this to mean that the scattering in the actual nebula is less important than in the assumed spherically symmetric model.

\section{RESULTS AND DISCUSSION}

Figure 1 shows our computed extinction curves (without scattering) superposed on the observed circumstellar dust 
extinction curve for $\alpha$ Sco, taken from Snow et al. (1987). The $\alpha$ Sco curve was originally derived by removing the foreground extinction, assuming that the foreground material is represented by the average interstellar extinction curve of Seaton (1979). When we have difficulty understanding how the circumstellar extinction could be negative at short UV wavelengths (the curve should flatten, not drop, as the parameter $2 \pi a / \lambda$ increases; e.g., Spitzer 1978), we decided to adopt as the assumed foreground extinction the curve for the $\rho$ Ophiuchi cloud, with which $\alpha$ Sco is closely associated. The $\rho$ Oph extinction is much lower than the average curve in the far-UV; the result of recomputing the $\alpha$ Sco circumstellar curve by subtracting a $\rho$ Oph-like foreground curve is indicated by the dots in Figure 1. The result is a flat far-UV curve that does not dip significantly below zero. Even though the far-UV extinction for a Sco dust has been revised by this, the original conclusions of Snow et al. (1987) need not be changed, because the far-UV extinction still shows no rise toward short wavelengths.

The smooth solid lines in Figure 1 represent our calculations without scattering, for a variety of assumed lower size cutoffs $a_{\min }$ (given on the plot, in units of microns). As these curves show, our computed curves for size distributions with small values of $a_{\min }$ are strongly ruled out by the observed a Sco curve. For $a_{\min }<800 \AA$, the calculated curves all rise toward short wavelengths (this trend is more pronounced for $a_{\min }<$ $250 \AA$, not shown in the figure). The best fit between computed and observed curves occurs for $a_{\min }=800 \AA$, and $a_{\min }=1000$ $\AA$ is within the uncertainties. Values as small as $a_{\min }=500 \AA$ or smaller are clearly ruled out.

Inclusion of scattering into the aperture does not significantly alter this result, as seen in Figure 2 . In fact, the scattering generally sharpens the argument for large grains in the circumstellar nebula around a Sco. The large silicate grains that produce the extinction at the $B$ and $V$ wavelengths are efficient scatters, with albedos above 0.8 and forwardscattering parameters greater than 0.5 (cf. Draine and Lee 1984). The inclusion of scattered photons has a large effect at these wavelengths: we find the effective $E(B-V)$ to be less than a fourth of the usual value. On the other hand, the small grains that produce the far-UV extinction are more absorbing even at these wavelengths, with albedos closer to 0.1 and nearly spherical scattering phase functions. Under these circumstances, very little light is scattered into the beam, and the effective extinction in the far-UV is only slightly less due to scattering. Dividing this by the much smaller effective $E(B-V)$ results in a greatly increased far-UV extinction in the normalized curve, in contradiction to the observations. Even with a lower size limit of $1000 \AA$, there is a significant rise in the extinction. Several different upper size limits on the grain population were also tested, without changing this result. We again conclude that scattering in the $\alpha$ Sco nebula cannot be as effective as in Code's symmetrical, centered circumstellar nebula. Since the inclusion of scattering raises the far-UV extinction level, the argument against any significant population of small grains in the circumstellar dust around $\alpha$ Sco is strengthened.

Our calculations therefore suggest that the lack of a significant rise in extinction toward short UV wavelengths indicates that there is no significant population of grains smaller than $800 \AA$ diameter. The size distributions of circumstellar grains in the observed stars must therefore cut off at a size larger than this; it is impossible to tell from our calculations how much larger. The conclusion that few or no grains smaller than $800 \AA$

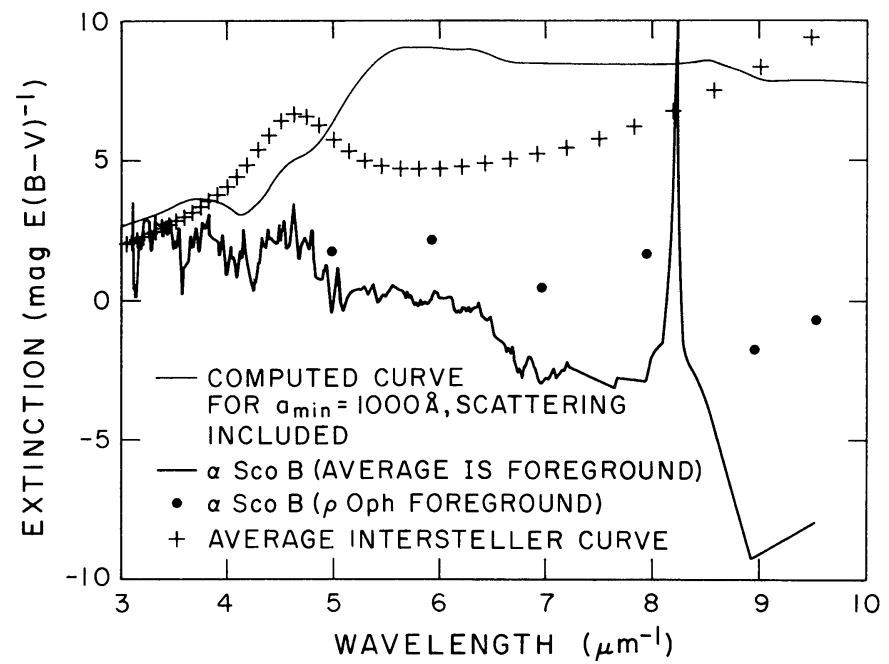

FIG. 2.-Circumstellar extinction of $\alpha$ Sco with the inclusion of scattering in the calculations. The thin line here represents the computed curve when scattering into the IUE aperture is taken into account. For this curve, the lower grain size limit was $1000 \AA$; the disagreement with observations becomes more severe if a smaller minimum size is assumed.

exist in these environments is consistent with some theoretical models for the grain-formation process (Hasegawa 1984), which indicate that newly formed grains grow rapidly as they flow outward from the central star, reaching sizes governed by how quickly they move to density regimes too sparse for continued accretion and growth. Other models (e.g., Gail and Sedlmayr 1986) are not consistent with our results.

Infrared polarization data (e.g., Hildebrand 1983) also suggest that large circumstellar grains are present in red supergiant extended atmospheres, although these data provide no information on the presence or absence of small grains.

Our data strongly support the theoretical expectations that newly formed circumstellar grains reach large sizes $(\sim 1 \mu \mathrm{m}$ or larger) before injection into the interstellar medium. On the other hand, there is ample evidence that small grains $(a \leq 250$ $\AA)$ are abundant in the interstellar medium, and a growing body of data suggests that there are numerous grains (or large molecules) with $\sim 10 \AA$ typical sizes (Sellgren 1984; Sellgren, Werner, and Dinerstein 1983). If these small grains are not formed in circumstellar envelopes of red giants, then we must look for other origins.

One possibility is that the small grains are formed in types of circumstellar environments not included in our study. It is particularly appealing to consider carbon-rich circumstellar envelopes, since most of the observable manifestations of small grains are generally attributed to carbonaceous grains. We are in the process of testing this possibility, through infrared searches in carbon-rich circumstellar environments for the spectral features associated with small $(\sim 10 \AA)$ carbonaceous grains or large molecules of the polycyclic aromatic hydrocarbon (PAH) class.

Another possible origin for the small grains assumed to be present in the general interstellar medium is the fragmentation of larger grains. The theoretical work on grain evolution by Seab and Shull (1985) and by Tielens et al. (1987) indicates that grains undergo shocks sufficiently frequently that shock processing should be the governing mechanism in establishing the size distribution. Our results may be construed as supporting this view. 
This research has been supported by NASA grant NSG5300 and NSF grant AST-8505587 to the University of Colorado. We thank Richard Buss for making available preliminary results of his work on ultraviolet circumstellar extinction lines.
We are also indebted to the Colorado Regional Data Analysis Facility for $I U E$ where the ultraviolet extinction curves were derived. Scattering models were run on the VAX cluster at the Computer Research Center of the University of New Orleans.

\section{REFERENCES}

Buss, R. H., and Snow, T. P. 1988, Ap. J., 335, 331.

Code, A. D. 1973, IAU Symposium 52, Interstellar Dust and Related Topics, ed

J. M. Greenberg and H. C. van de Hulst (Dordrecht: Reidel), p. 505.

Draine, B. T. 1985, Ap. J. Suppl., 57, 587

Draine. 1987, unpublished.

Draine, B. T., and Lee, H. M. 1984, Ap. J., 285, 89.

Draine, B. T., and Sutin, B. 1987, Ap. J., 320, 803.

Gail, H.-P., and Sedlmayr, E. 1986, Astr. Ap., 166, 225.

Hasegawa, H. 1984, in Laboratory and Observationsl Infrared Spectra of Interstellar Dust, ed. R. D. Wolstencroft and J. M. Greenberg (Edinburgh: Royal Observatory), p. 36.

Hecht, J. H. 1986, Ap. J., 305, 817.

Hildebrand, R. H. 1983, Quart. J. R.A.S., 24, 267.

Jura, M. 1976, Ap. J., 204, 12.

Mathis, J. S., Rumpl, W., and Nordsieck, K. H. 1977, Ap. J., 217, 425.

Mathis, J. S., and Wallenhorst, S. G. 1981, Ap. J., 244, 483.

McKee, C. F., Hollenbach, D. J., Seab, C. G., and Tielens, A. G. G. M. 1987, Ap. J., 318, 674 .

Sakata, A., Wada, S., Tanabe, T., and Onaka, T. 1984, Ap. J. (Letters), 287, L51. Savage, B. D., and Mathis, J. 1979, Ann. Rev. Astr. Ap., 17, 73.

Seab, C. G. 1987, in Interstellar Processes, ed. D. J. Hollenbach and H. A. Thronson (Dordrecht: Reidel), p. 491.

Seab, C. G., and Shull, J. M. 1983, Ap. J., 275, 652.

Seaton, M. J. 1979, M.N.R.A.S., 187, 73P.

Sellgren, K. 1984, Ap. J., $277,623$.

Sellgren, K., Werner, M., and Dinerstein, H. 1983, Ap. J. (Letters), 271 , L13.

Snow, T. P., Buss, R. H., Gilra, D. P., and Swings, J. P. 1987, Ap. J., 321, 921

Tielens, A. G. G. M., Seab, C. G., Hollenbach, D. J., and McKee, C. F. 1987, Ap. J. (Letters), 319, L109.

van de Hulst, H. C. 1957, Light Scattering by Small Particles (New York: Wiley).

Witt, A. N. 1973, Interstellar Dust and Related Topics, ed. J. M. Greenberg and H. C. van de Hulst (Dordrecht: Reidel), p. 53.

C. Gregory Seab: Department of Physics, University of New Orleans, Lakefront, New Orleans, LA 70148

Theodore P. SNOw: Center for Astrophysics and Space Astronomy, University of Colorado, Boulder, CO 80309 